\title{
Long noncoding RNA uc003pxg.1 regulates endothelial cell proliferation and migration via miR-25-5p in coronary artery disease
}

\author{
PING LI $^{1}$, YUAN LI $^{2}$, LU CHEN $^{2}$, XUEXING MA $^{2}$, XINXIN YAN $^{1}$, MEINA YAN $^{1}$, BUYUN QIAN $^{2}$, \\ FENG WANG ${ }^{1}$, JINGYI XU ${ }^{1}$, JUAN YIN $^{1}$, GUIDONG XU ${ }^{2}$ and KANGYUN SUN ${ }^{2}$ \\ Departments of ${ }^{1}$ Central Laboratory and ${ }^{2}$ Cardiology, The Affiliated Suzhou Hospital of Nanjing Medical University, \\ Suzhou Municipal Hospital, Gusu School, Nanjing Medical University, Suzhou, Jiangsu 215008, P.R. China
}

Received January 19, 2021; Accepted May 21, 2021

DOI: $10.3892 / \mathrm{ijmm} .2021 .4993$

\begin{abstract}
Long noncoding RNAs (IncRNAs) have been reported to be associated with the progression of coronary artery disease (CAD). In our previous study, the levels of lncRNA uc003pxg.l were upregulated in patients with CAD compared with those in control subjects. However, the role and underlying mechanism of the effects of uc003pxg.1 in CAD remain unknown. Therefore, the aim of the present study was to investigate the expression pattern and biological function of uc003pxg.1 in CAD. First, uc003pxg.1 expression levels were assessed in peripheral blood mononuclear cells isolated from patients with CAD by reverse transcription-quantitative (RT-q)PCR. The results demonstrated that the levels of uc003pxg.l were significantly upregulated ( 4.6-fold) in samples from 80 patients with CAD compared with those in 80 healthy subjects. Subsequently, the present study demonstrated that small interfering RNA-mediated uc003pxg.l knockdown inhibited human umbilical vein endothelial cell (HUVEC) proliferation and migration, which was analyzed using the Cell Counting Kit-8, cell cycle, EdU and Transwell assays. Additionally, the results of RT-qPCR and western blot analyses revealed that uc003pxg.1 regulated the mRNA and protein levels of cyclin D1 and cyclin-dependent kinase. Through high-throughput sequencing and dual-luciferase reporter assays, the present study demonstrated that microRNA (miR)-25-5p was a downstream target of uc003pxg.1. Further experiments verified that $u c 003 p x g .1$ regulated HUVEC proliferation and migration via $m i R-25-5 p$. The results of the
\end{abstract}

Correspondence to: Dr Kangyun Sun or Dr Guidong Xu, Department of Cardiology, The Affiliated Suzhou Hospital of Nanjing Medical University, Suzhou Municipal Hospital, Gusu School, Nanjing Medical University, 242 Guangji Road, Suzhou, Jiangsu 215008, P.R. China

E-mail: skywj66@163.com

E-mail: huatuo02014@126.com

Key words: long noncoding RNA, coronary artery disease, peripheral blood mononuclear cells, cell proliferation and migration present study may enhance the current understanding of the role of lncRNA uc003pxg.1 in CAD.

\section{Introduction}

Coronary artery disease (CAD) is the most common type of organ lesion caused by atherosclerosis and affects the life quality of patients (1). Atherosclerosis is a major issue globally, since an estimated $31 \%$ of deaths worldwide are caused by cardiovascular disease, and the number is steadily increasing (2). The cause of CAD remains incompletely understood, as it may be the result of a combination of multiple factors. Risk factors for CAD include age, sex, hypertension, diabetes mellitus, smoking, dyslipidemia and obesity (3). Currently, effective treatment strategies include secondary prevention, plasma oxidized low-density lipoprotein (LDL) reduction, antiplatelet agents, anticoagulants, blood pressure and diet control, exercise and percutaneous coronary intervention, or coronary artery bypass surgery selectively when the major coronary artery exhibits $>50 \%$ stenosis (4). However, the mortality rate of AMI remains high due to the difficulty of diagnosing CAD at early stages, and the development of new noninvasive methods for the early diagnosis of CAD is crucial for improving its prognosis (5). Since the molecular mechanisms underlying these pathological and pathophysiological processes are not completely understood, the identification of key regulatory molecules of cardiovascular disease is necessary to develop effective preventive measures and treatments (6).

It has been demonstrated that $>75 \%$ of the human genome encodes noncoding RNAs (ncRNAs). Long noncoding RNAs (lncRNAs; ncRNAs >200 nucleotides) have emerged as notable contributors in the regulation of chromatin modification, gene expression, cell proliferation, differentiation and human diseases such as cancer, neurological disorders and immunological disorders $(7,8)$. The expression patterns, tumor specificity and stability of lncRNAs in circulating body fluids (plasma and urine) have provided a basis for the diagnosis and treatment of cancer $(9,10)$. Previous studies have demonstrated that IncRNAs are involved in the development of various CADs through the regulation of cell differentiation, proliferation, apoptosis, necrosis and autophagy (11-13). 
In addition, IncRNAs and microRNAs (miRNAs) regulate the initiation and progression of CAD by regulating various molecular mechanisms (6). For example, lncRNA nuclear enriched abundant transcript 1 increases cell viability and affects CAD by activating miR-140-3p (14). High plasma levels of $H 19$ and mitochondrial lncRNA uc022bqs.l are associated with a high risk of CAD, and these molecules have the potential to become novel biomarkers of CAD (15). The interaction of metastasis-associated lung adenocarcinoma transcript 1 (MALAT1), $m i R-22$ and $C X C R 2 / A K T$ in the regulation of endothelial cell function demonstrates the complex roles of IncRNA-miRNA-mRNA cascades in the progression of CAD $(16,17)$.

Previous studies have also revealed that cell proliferation and migration are associated with the occurrence and development of CAD. For example, variations in 9p21 affect the expression of cyclin-dependent kinase $2 \mathrm{~A}(C D K N 2 A)$ and $2 \mathrm{~B}$ $(C D K N 2 B)$ in vascular smooth muscle cells (VSMCs) as well as their proliferation, which may be an important mechanism underlying the association between 9p21 and CAD susceptibility (18). In vascular cells, T-cadherin (T-CAD) promotes cell proliferation by regulating the cell cycle, indicating that this protein may promote the progression of proliferative vascular diseases, such as atherosclerosis, restenosis and tumor angiogenesis (19). Downregulation of MALAT1 is associated with numerous pathological processes, including CAD, as it inhibits the proliferation of vascular endothelial cells and VSMCs, reduces myocardial cell apoptosis and improves left ventricular function (20). In addition, the lncRNA maternally expressed gene 3 suppresses endothelial cell proliferation and migration by regulating $m i R-21$ (21).

In our previous study, a previously unreported lncRNA uc003pxg.1 was identified (22); the genomic location and sequence of uc003pxg.1 are presented in Data S1. The aim of the present study was to determine whether the expression levels of uc003pxg.1 differed in peripheral blood mononuclear cells (PBMCs) isolated from patients with CAD and healthy control subjects, and to evaluate the interactions between miRNAs and uc003pxg.1 in human umbilical vein endothelial cells (HUVECs).

\section{Materials and methods}

Study subjects. Between August 2017 and December 2019 , 160 subjects ( 80 patients with CAD and 80 controls) aged 40-85 years were enrolled in the present study at the Department of Cardiology of The Affiliated Suzhou Hospital of Nanjing Medical University (Suzhou, China). CAD was confirmed by angiographic evidence in at least one segment of a major coronary artery, including the left anterior descending artery, left circumflex artery or right coronary artery, with $>50 \%$ stenosis (23). Patients with severe primary disease, acute infection, trauma, myocardial infarction, cerebral infarction, cancer and alcohol or drug addiction were excluded from the study. The 80 control subjects were selected from individuals undergoing routine health examinations. The reference intervals of the routine blood tests were as follows: Blood pressure, 90-140/60-90 mmHg; blood glucose, 4-6.1 mmol/1; total cholesterol, 2.8-5.17 mmol/1; triglycerides, 0.56-1.7 mmol/1; and cholesterol lipids, 2.8-5.17 $\mathrm{mmol} / 1$. The experiments conducted in the present study were approved by the Ethics Committee of Nanjing Medical University (KL901117). Written informed consent was obtained from all patients or their families.

Cell culture and transfection. Immortalized HUVECs (cat. no. CW2919S) were purchased from CoWin Biosciences and cultured in DMEM (HyClone; Cytiva) containing 10\% fetal bovine serum (FBS, Gibco; Thermo Fisher Scientific, Inc.), $100 \mathrm{U} / \mathrm{ml}$ streptomycin and $100 \mathrm{U} / \mathrm{ml}$ penicillin at $37^{\circ} \mathrm{C}$ in a humidified incubator under an atmosphere with $5 \% \mathrm{CO}_{2}$. Prior to use, the cells were washed with phosphate-buffered saline (PBS; HyClone; Cytiva) and digested with a Trypsin-EDTA solution (MilliporeSigma).

For transfection, HUVECs were seeded at $\sim 50 \%$ confluence in a 24 -well at $24 \mathrm{~h}$ pre-transfection. Subsequently, the cells were transfected with $100 \mathrm{nM}$ small interfering (si)RNA targeting lncRNA uc003pxg.1 (si-uc003pxg.1), negative control (NC) siRNA (si-NC), mir-25-5p mimic, mir-25-5p inhibitor or corresponding NC (Guangzhou RiboBio Co., Ltd.) using a ribo $F E C T^{\text {тм }} \mathrm{CP}$ Transfection kit (Guangzhou RiboBio Co., Ltd.). A total of $2.5 \mu \mathrm{l}$ siRNA was diluted with $30 \mu \mathrm{l} 1 \mathrm{X}$ riboFECT $T^{\mathrm{TM}} \mathrm{CP}$ Buffer and gently mixed. Subsequently, $3 \mu \mathrm{l}$ ribo $F E C T^{\mathrm{TM}} \mathrm{CP}$ Reagent was added and mixed gently, incubated for $\leq 15 \mathrm{~min}$ at room temperature, and the transfection complexes were added into the medium and incubated in an incubator for $24-72 \mathrm{~h}$ at $37^{\circ} \mathrm{C}$ with $5 \% \mathrm{CO}_{2}$. The cells were collected at 24 and $48 \mathrm{~h}$ post-transfection for use in reverse transcription-quantitative (RT-q)PCR and western blotting analyses, respectively. The oligomer sequences were as follows: si-uc003pxg.1, 5'-GCAATGTAGTCACCAATAA-3'; si-NC, 5'-GGCTCTAGAAAAGCCTATGC-3'; miRNA mimic NC, 5'-UUUGUACUACACAAAAGUACUG-3'; miRNA inhibitor NC, 5'-CAGUACUUUUGUGUAGUACAAA-3'; has-miR-25-5p mimics sense, 5'-AGGCGGAGACUUGGG CAAUUG-3' and antisense, 5'-CAAUUGCCCAAGUCUCCG CCU-3'; has-miR-25-5p inhibitor, 5'-CAAUUGCCCAAG UCUCCGCCU-3'.

PBMC isolation, RNA isolation and RT-qPCR. Blood samples (4-6 $\mathrm{ml})$ from each participant were collected in EDTA vacuum anticoagulant blood vessels. The plasma was collected following centrifugation at $1,600 \mathrm{x} \mathrm{g}$ for $10 \mathrm{~min}$ at $4^{\circ} \mathrm{C}$ to remove the debris. PBMCs were isolated from the blood cells via Ficoll-Paque PLUS (GE Healthcare) density-gradient centrifugation as previously described $(24,25)$ and washed twice with saline.

Total RNA was extracted from PBMCs and HUVECs with TRIzol ${ }^{\circledR}$ Reagent (Ambion; Thermo Fisher Scientific, Inc.). Reverse transcription of $500 \mathrm{ng}$ of RNA into cDNA was performed with $2 \mu \mathrm{l} 5 \mathrm{X}$ PrimeScript ${ }^{\mathrm{TM}}$ RT Master Mix (cat. no. RR036A; Takara Bio, Inc.), and the reaction was adjusted to $10 \mu \mathrm{l}$ with RNase-free $\mathrm{dH}_{2} \mathrm{O}$. The thermocycling conditions were as follows: $37^{\circ} \mathrm{C}$ for $15 \mathrm{~min} ; 85^{\circ} \mathrm{C}$ for $5 \mathrm{sec}$; and hold at $4^{\circ} \mathrm{C}$. RT-qPCR was performed using SYBR ${ }^{\circledR}$ Premix Ex Taq $^{\text {TM }}$ II (Takara Bio, Inc.) on a LightCycler ${ }^{\circledR} 480$ Instrument II Real-Time PCR Detection system (Roche Diagnostics), and $\beta$-actin was used as the internal reference. The thermocycling conditions were as follows: $95^{\circ} \mathrm{C}$ for $30 \mathrm{sec}$; 40 cycles of $95^{\circ} \mathrm{C}$ for $5 \mathrm{sec}$ and $60^{\circ} \mathrm{C}$ for $20 \mathrm{sec}$; and $65^{\circ} \mathrm{C}$ for $15 \mathrm{sec}$. The primers 
used in this experiment were as follows: uc003pxg.l forward, 5'-GTTACCAGAAAGCGTTGCCA-3' and reverse, 5'-TAT ACTCAGTCCAGCAGCCC-3'; cyclin D1 forward, 5'-GCT GCGAAGTGGAAACCATC-3' and reverse, 5'-CCTCCTTCT GCACACATTTGAA-3'; and CDK6 forward, 5'-CATACC CTCTCTGCTGCTTT-3' and reverse, 5'-TGCTACTCATTT TGCTCACCT-3'; $\beta$-actin forward, 5'-CACGAAACTACC TTCAACTCC-3' and reverse, 5'-CATACTCCTGCTTGCTGA TC-3' and were synthesized by Genewiz, Inc. For miRNA quantification, bulge-loop RT primers and qPCR primers specific for $m i R-25-5 p$ were designed and synthesized by Guangzhou RiboBio Co., Ltd. (product no. MQPS0000882-1-100; RT $m i R-25-5 p$, cat. no. ssD809231465; $m i R-25-5 p$ forward, cat. no. ssD809231628; and $m i R-25-5 p$ reverse, cat. no. ssD089261711); U6 (Bulge-Loop U6 qPCR Primer Set; cat. no. MQPS0000002-1-100) was used as the internal control, and the experiments were performed in triplicate. The relative expression data were analyzed using the $2^{-\Delta \Delta \mathrm{Cq}}$ method (26) following normalization to the $\beta$-actin $(A C T B)$ or $U 6$ levels.

High-content screening (HCS). The differentially expressed IncRNAs ENST00000583122, ENST00000606540, NR_027387, uc003pxg.1, ENST00000602845, ENST00000601618 were selected from the 1ncRNA-seq (accession no. GSE171551). siRNA mixes of these lncRNAs were designed and synthesized by RiboBio Co., Ltd. EdU staining was performed after 100-nM lncRNA siRNA mixes were transfected into adherent HUVECs. The siRNA mix sequences were as follows: si-ENST00000583122, 5'-AGA GATACA AGA AGGTCA A-3', 5'-CCGACA AAA TGG GCTTAAT-3' and 5'-CAGATGTCACAATGTGATA-3'; si-ENST00000606540, 5'-AGGCGA AACTGTCCTCA AA-3', 5'-CTCCCAATTTGGAAAGTA A-3' and 5'-GGA GAGGCTGATTTGAAAA-3'; si-NR_027387, 5'-TGACCA ACTCATCTACTCT-3', 5'-ATCACTCCTTGACCATTAT-3' and 5'-GCAAATGTCATGTTCTGTT-3'; si-uc003pxg.1, 5'-GCAATGTAGTCACCAATAA-3', 5'-CACTAACCTACA ACCTTCA-3' and 5'-CCAAGCAATAACCCTTGTA-3'; si-ENST00000602845, 5'-CCCTGGCGCAAACATTTAA-3', 5'-AGCGATTTAGAGACGCCTT-3' and 5'-CGATTTAGA GACGCCTTCA-3'; and si-ENST00000601618, 5'-GCGAAA TGCTTGACAATCA-3', 5'-TGCTTGACAATCACAATCA-3' and 5'-TGACAGCCAGTAGCATTTA-3'. Subsequently, the cells were rapidly detected with the GE IN CELL Analyzer $6500 \mathrm{HS}$ high-content screening platform (Cytiva) by Guangzhou RiboBio Co., Ltd.. The cell proliferation rate was calculated based on the proportion of proliferating cells among the total cells, and functional target lncRNAs were identified based on differences in proliferation.

miRNA-seq high-throughput sequencing and the selection of target miRNA. High-throughput sequencing was performed by Guangzhou RiboBio Co., Ltd. siRNA (100 nM) was transfected into HUVECs, and RNA was extracted from HUVECS in the NC and si-uc003pxg.1 groups using the MagZol (Magen BioSciences). The quantity and integrity of the RNA yield were assessed by K5500 and the Agilent 2200 TapeStation (Agilent Technologies, Inc.). The total RNAs were ligated with a 3 ' RNA adaptor, and followed by 5 ' adaptor ligation. Subsequently, the adapter-ligated RNAs were subjected to
RT-PCR and amplified with a low cycle: Initial denaturation at $94^{\circ} \mathrm{C}$ for $30 \mathrm{sec} ; 12-15$ cycles of denaturation at $94^{\circ} \mathrm{C}$ for $15 \mathrm{sec}$, annealing at $62^{\circ} \mathrm{C}$ for $30 \mathrm{sec}$ and extension at $70^{\circ} \mathrm{C}$ for $15 \mathrm{sec}$; final extension at $70^{\circ} \mathrm{C}$ for $5 \mathrm{~min}$; hold at $4^{\circ} \mathrm{C}$. The PCR products were size-selected by PAGE. The adaptors, primers, enzymes and buffers required to convert small RNAs into indexed libraries for next-generation sequencing on the Illumina platform were included in the NEBNext ${ }^{\circledR}$ Multiplex Small RNA Library Prep Set for Illumina ${ }^{\circledR}$ (Index Primers 1-48, cat. no. NEB \#E7560S; Illumina, Inc.), and all procedures were performed according to the manufacturer's instructions. The purified library products were evaluated using an Agilent 2200 TapeStation. The libraries were sequenced on a HiSeq 2500 instrument (Illumina, Inc.) to generate single-end 50-bp reads by Guangzhou RiboBio Co., Ltd.. The miRNA expression was calculated as the reads per million (RPM) values as follows: $\mathrm{RPM}=$ (no. of reads mapping to $\mathrm{miRNA} /$ no. of reads in clean 50 data) $\times 10^{6}$. The expression levels were calculated, and the miRNA expression clusters were analyzed to identify miRNAs that were differentially expressed. The expression of miRNAs were verified by RT-qPCR in 25 patients with CAD and 25 control subjects selected from the 160 clinical samples.

RNA fluorescence in situ hybridization (FISH). HUVECs were cultured in 24-well plates with round coverslips at the bottom overnight. FISH assays were performed using a Ribo ${ }^{\mathrm{TM}}$ Fluorescent In Situ Hybridization kit (Guangzhou RiboBio Co., Ltd.). The Cy 3-labeled DNA probes of uc003pxg.1, 18S and U6 (RiboTMh-lnc uc003_FISH-probe Mix; product no. Inc1101634; Red, 20T) were designed by Guangzhou RiboBio Co., Ltd.. After the cells reached $60-70 \%$ confluence, they were washed with PBS and fixed with $4 \%$ paraformaldehyde for $10 \mathrm{~min}$ at room temperature. Subsequently, $1 \mathrm{ml}$ precooled permeabilization solution was added to each well and incubated for $5 \mathrm{~min}$ at $4^{\circ} \mathrm{C}$. Following washing with PBS, $200 \mu \mathrm{l}$ prehybridization solution was added to each well and incubated at $37^{\circ} \mathrm{C}$ for $30 \mathrm{~min}$. Then, $100 \mu \mathrm{l}$ probe hybridization solution was added to each well and hybridized overnight at $37^{\circ} \mathrm{C}$ in the dark. The cells were washed with saline sodium citrate and PBS, and DAPI was added and incubated for $10 \mathrm{~min}$ at room temperature. Images $(\geq 3)$ were captured using confocal fluorescence microscopy at x63 magnification.

Dual-luciferase reporter assay. A uc003pxg.1 fragment containing the $m i R-25-5 p$-binding site was constructed and cloned into the target pmirGLO dual-luciferase reporter gene vector (Guangzhou RiboBio Co., Ltd.) to generate the construct pmirGLO-uc003pxg. l-wild-type (wt). A uc003pxg.1 sequence containing a mutated $m i R-25-5 p$-binding site was cloned into the vector to generate the construct pmirGLO-uc003pxg.1-mutant (mut). The binding target prediction of miR-25-5p and uc003pxg. 1 are presented in Data S1. The vectors were transfected into $E$. coli DH5 $\alpha$ competent cells (cat. no. CS01010, Anhui General Biosystems Co., Ltd.), cultured overnight at $37^{\circ} \mathrm{C}$, and the plasmids were extracted from the positive clones. The $m i R-25-5 p$ mimics and $m i R-N C$ were transfected into HUVECs, which were subsequently transfected with pmirGLO-uc003pxg.1-wt or pmirGLO-uc003pxg.1-mut. HUVECs were seeded in 6-well plate the day before transfection. A total of $2 \mu \mathrm{g}$ plasmid and 
or miRNAs were diluted with $100 \mu 1$ OPTI-MEM medium and incubated for $5 \mathrm{~min}$ at room temperature. Subsequently, Lipofectamine ${ }^{\circledR} 3000$ (Invitrogen; Thermo Fisher Scientific, Inc.) was added to the diluted plasmids, and X-tremeGENE siRNA Transfection Reagent (Guangzhou RiboBio Co., Ltd.) was added to the diluted miRNAs, incubated for $15 \mathrm{~min}$ at room temperature, added into 6-well plates and incubated in an incubator at $37^{\circ} \mathrm{C}$ with $5 \% \mathrm{CO}_{2}$. The luciferase activity was measured at $24 \mathrm{~h}$ post-transfection with a Dual-Luciferase Reporter Assay system (cat. no. E1910; Promega Corporation), and the firefly luciferase activity relative to Renilla luciferase activity for each construct was compared with that of the control group.

Cell viability. Cell Counting Kit-8 (CCK-8; Dojindo Laboratories, Inc.) was used to assess cell proliferation. At 12-24 h post-transfection with $100 \mathrm{nM}$ siRNA, 1,000 HUVECs (100 $\mu \mathrm{l}$ cell suspension) were seeded in each well of a 96-well plate and cultured in an incubator for 3-4 h until the cells adhered to the well. Subsequently, $10 \mu 1$ CCK- 8 solution was added to each well every $24 \mathrm{~h}$ for 3 days and incubated in an incubator at $37^{\circ} \mathrm{C}$ under an atmosphere with $5 \% \mathrm{CO}_{2}$ for $2-4 \mathrm{~h}$. Absorbance at $450 \mathrm{~nm}$ was determined by a microplate reader.

Cell cycle analysis. At $48 \mathrm{~h}$ post-transfection, $\sim 5 \times 10^{4}$ adherent HUVECs in 6-well plates were digested with trypsin and washed with PBS. Then, the cells were fixed with $70 \%$ cold ethanol for $>24 \mathrm{~h}$ at $-20^{\circ} \mathrm{C}$, after which they were treated with $10 \mu 1$ RNase A and $25 \mu 1$ propidium using a Cell Cycle and Apoptosis Analysis kit (Beyotime Institute of Biotechnology) for $30 \mathrm{~min}$ at $37^{\circ} \mathrm{C}$. Subsequently, the cells were washed with cold PBS, and fluorescence at $488 \mathrm{~nm}$ was detected by flow cytometry (BD FACSCalibur ${ }^{\mathrm{TM}}$; Becton Dickinson and Company). The data were analyzed using FlowJo V7.6 software (BD Biosciences).

5-Ethynyl-2'-deoxyuridine (EdU) assay. A Cell-Light EdU Apollo 567 In Vitro Flow Cytometry kit (Guangzhou RiboBio Co., Ltd.) was used for the EdU assay. HUVECs were seeded at 40-60\% confluence in 24-well plates with round coverslips and incubated overnight. Subsequently, at $48 \mathrm{~h}$ post-transfection with $100 \mathrm{nM}$ siRNA, the cells were washed with PBS, fixed with $4 \%$ paraformaldehyde for $30 \mathrm{~min}$, and then treated with a $0.5 \%$ Triton $\mathrm{X}-100 / \mathrm{PBS}$ solution for $10 \mathrm{~min}$ at room temperature. The cells were incubated with $1 \mathrm{X}$ Apollo reagent for $30 \mathrm{~min}$ in the dark at room temperature according to the manufacturer's instructions, followed by staining with $1 \mathrm{X}$ Hoechst for $30 \mathrm{~min}$ at room temperature. Images ( $\geq 3$ per sample) were captured by confocal fluorescence microscopy at x40 magnification (LSM900; Zeiss AG). The percentage of EdU-positive cells was quantified using ImageJ v1.8.0 (National Institutes of Health).

Transwell assay. For the Transwell migration assays, 24-well 8.0- $\mu \mathrm{m}$ pore membranes (Corning, Inc.) were used. At $24 \mathrm{~h}$ post-transfection with $100 \mathrm{nM}$ siRNA, $4 \times 10^{4}$ cells were seeded in the upper chamber with $200 \mu \mathrm{l}$ serum-free medium, and 600-800 $\mu \mathrm{l}$ culture medium containing 10\% FBS (Gibco; Thermo Fisher Scientific, Inc.) was added to the lower chamber. Following a $24 \mathrm{~h}$ incubation at $37^{\circ} \mathrm{C}$, the cells on the upper surface of the membrane were gently removed with cotton swabs, and the cells on the lower surface were fixed with $4 \%$ paraformaldehyde for $30 \mathrm{~min}$ and stained with $800 \mu \mathrm{l}$ crystal violet solution for $15-30 \mathrm{~min}$ at room temperature. The cells that passed through the membranes were imaged in $\geq 3$ fields of view with an inverted fluorescence microscope at x20 magnification (Leica Microsystems $\mathrm{GmbH}$ ).

Western blotting. At $48 \mathrm{~h}$ post-transfection with $100 \mathrm{nM}$ siRNA in a 6-well plate, proteins were extracted from HUVECs using RIPA buffer (Beyotime Institute of Biotechnology) supplemented with protease inhibitors. The protein concentrations were determined with an Ultramicro Nucleic Acid Analyzer (Hangzhou Allsheng Instruments Co., Ltd.), and 20-40 $\mu \mathrm{g}$ protein/lane was separated using $10 \%$ SDS-polyacrylamide gels by electrophoresis, following which the proteins were transferred to $0.2-\mu \mathrm{m}$ Immobilon ${ }^{\circledR}-\mathrm{P}^{\mathrm{SQ}} \mathrm{PVDF}$ membranes. Subsequently, the membranes were blocked with $5 \%$ skim milk and incubated with rabbit anti-cyclin D1 (1:1,000; cat. no. 2978T), anti-cyclin B1 (1:1,000; cat. no. 4138T), anti-CDK4 (1:1,000; cat. no. 12790T), anti-CDK6 (1:1,000; cat. no. 1333T), anti-proliferating cell nuclear antigen (PCNA; 1:1,000; cat. no. 13110T), anti-vimentin (1:1,000; cat. no. 5741T), mouse anti-cyclin E1 (1:1,000; cat. no. 4129T) (all Cell Signaling Technology, Inc.) and anti-GAPDH (1:5,000; cat. no. 60004-1-lg; Proteintech Group, Inc.) antibodies overnight at $4^{\circ} \mathrm{C}$. The goat anti-mouse $\operatorname{lgG}(\mathrm{H}+\mathrm{L})$ (1:50,000; cat. no. A00615) and goat anti-rabbit $\lg \mathrm{G}(\mathrm{H}+\mathrm{L})$ (1:50,000; cat. no. A00834) secondary antibodies (both Multisciences Biotech Co., Ltd.) were diluted with 5\% skim milk and added to the membranes for $1 \mathrm{~h}$ at room temperature. Following washing the membrane three times for 10 min each with TBS- $0.1 \%$ Tween-20 washing buffer, the expression levels of the proteins were detected with an automatic Tanon 5200 Multi chemiluminescence image analyzer using Tanon ${ }^{\mathrm{TM}}$ High-sig ECL western blotting Substrate (both Tanon Science and Technology Co., Ltd.). The gray area was determined using ImageJ v1.8.0 (National Institutes of Health).

Bioinformatics analysis. The original sequencing data of IncRNA-seq from our previous study (22) and miRNA-seq from the current study were uploaded to the GEO database of NCBI (accession nos. IncRNA-seq, GSE171551; https://www.ncbi. nlm.nih.gov/geo/query/acc.cgi?acc $=$ GSE171551; miRNA-seq, GSE171715; https://www.ncbi.nlm.nih.gov/geo/query/acc. cgi?acc=GSE171715). The upregulated miRNAs were selected with P-value $<0.05$ and fold-change $>1$. Differential expression between two sets of miRNA-seq samples was calculated with the 'DEseq (V 1.32.0)' algorithm (27) according to the criteria of $\mid \log 2$ (fold change) $\mid \geq 1$ and P-value $<0.05$. miRNA target genes were predicted, TargetScan, miRDB (http://mirdb.org), miRTarBase (http://mirtarbase.mbc.nctu. edu.tw/) and miRWalk (http://zmf.umm.uni-heidelberg. de/apps/zmf/mirwalk/) were used to predict the target genes of the selected miRNAs. Gene ontology (GO) and Kyoto Encyclopedia of Genes and Genomes (KEGG) pathway enrichment analyses were performed using the target genes and KOBAS (http://kobas.cbi.pku.edu.cn/kobas3) was used for GO and KEGG pathway analyses. The target genes involved in KEGG analyses are listed in Table SI. miRanda (http://www. 
Table I. Clinical characteristics of the study subjects.

\begin{tabular}{|c|c|c|c|}
\hline Variable & CAD $(n=80)$ & $\mathrm{CON}(\mathrm{n}=80)$ & P-value \\
\hline Age, years & $67.4 \pm 17.6$ & $62.5 \pm 18.5$ & 0.786 \\
\hline Sex, male, n (\%) & $48(60.0 \%)$ & $45(56.3 \%)$ & 0.374 \\
\hline $\mathrm{BMI}, \mathrm{kg} / \mathrm{m}^{2}$ & $25.39 \pm 3.72$ & $25.68 \pm 3.13$ & 0.916 \\
\hline Smoking, n (\%) & $30(37.5 \%)$ & $15(18.8 \%)$ & 0.119 \\
\hline Drinking, n (\%) & $12(15.0 \%)$ & $10(12.5 \%)$ & 0.409 \\
\hline Diabetes, n (\%) & $29(36.3 \%)$ & $12(15.0 \%)$ & $0.002^{\mathrm{a}}$ \\
\hline Hypertension, n (\%) & $69(86.3 \%)$ & $26(32.5 \%)$ & $<0.001^{\mathrm{a}}$ \\
\hline $\mathrm{TC}, \mathrm{mmol} / \mathrm{l}$ & 4.27 & 4.33 & 0.731 \\
\hline $\mathrm{TG}, \mathrm{mmol} / \mathrm{l}$ & 1.66 & 1.68 & 0.930 \\
\hline HDL, mmol/1 & 1.04 & 1.12 & 0.090 \\
\hline LDL, mmol/1 & 2.64 & 2.70 & 0.616 \\
\hline VLDL, mmol/1 & 0.67 & 0.52 & 0.107 \\
\hline
\end{tabular}

${ }^{\mathrm{a}} \mathrm{P}<0.05$. CAD, coronary artery disease; CON, control; TC, total cholesterol; TG, triglyceride; HDL, high-density lipoprotein; LDL, low-density lipoprotein; VLDL, very low-density lipoprotein.

microrna.org/microrna/home.do) was used for the binding target prediction of $m i R-25-5 p$ and $u c 003 p x g .1$, whereas TargetScan (http://www.targetscan.org/vert_71/) was used to predict the target genes of miRNAs.

Statistical analyses. Statistical analysis was conducted using SPSS 21.0 (IBM Corp.), and GraphPad Prism 7 (GraphPad Software, Inc.) was used for data analysis. Data are presented as the mean \pm SD of three independent experiments. Two-tailed Student's t-test was used for comparison between two groups of normally distributed variables, and one-way ANOVA with Tukey's post-hoc test was performed for analysis among multiple groups. Discrete variables were compared by contingency table analysis of the $\chi^{2}$ test. Receiver operating characteristic (ROC) curves and the area under the ROC curve (AUC) were used to assess the feasibility of uc003pxg.1 as a biomarker for the diagnosis of $\mathrm{CAD}$. $\mathrm{P}<0.05$ was considered to indicate a statistically significant difference.

\section{Results}

Verification of uc003pxg.1 expression in clinical samples and statistical analysis of clinical indicators. PBMCs from 80 patients with CAD and 80 controls were isolated for analysis. The clinicopathological characteristics of the patients and controls are listed in Table I. The hypertension (86.3\%), diabetes $(36.3 \%)$ and smoking $(37.5 \%)$ rates were significantly higher in patients with CAD compared with those in the control group, and the HDL levels exhibited a decreasing but not significant trend in the CAD group compared to the control group.

High-content screening, selection and cellular localization of target lncRNAs. The lncRNA uc003pxg.1 was discovered by IncRNA-seq high-throughput sequencing performed in our previous study (22). In the present study, the relative expression levels of uc003pxg.1 in PBMCs from 80 patients with CAD and 80 controls were determined by RT-qPCR. The results demonstrated that uc003pxg.1 was significantly upregulated ( 4.6-fold) in PBMCs isolated from patients with CAD compared with the control group ( $\mathrm{P}<0.01$; Fig. 1A), and the area under the ROC curve was 0.7163 (Fig. 1B), suggesting that uc003pxg.1 may be a potential biomarker for the clinical diagnosis of CAD. A total of six lncRNAs were selected for cell proliferation high-content screening to identify lncRNAs associated with biological functions. The percentage of EdU-positive cells in the si-uc003pxg.1-transfected group was significantly decreased compared with that in the control-transfected group (Figs. 1C and S1), indicating that uc003pxg.1 may serve biological functions in cell proliferation. The FISH results demonstrates that uc003pxg.1 was primarily localized to the nucleus (Fig. 1D), with a limited amount detected in the cytoplasm. The competing endogenous RNA regulatory mechanism is involved in the post-transcriptional regulation in the cytoplasm (28); thus, cytoplasmic uc003pxg.1 was used for the subsequent competing endogenous RNA network analysis.

uc003pxg.1 promotes cell proliferation and migration. Following HUVEC transfection with si-uc003pxg.1, cell proliferation was significantly inhibited, as demonstrated by the results of the EdU (Fig. 2A), CCK-8 (Fig. 2B) and cell cycle (Fig. 2C) assays. Transwell assay results also revealed that the migratory ability of the cells in the si-uc003pxg.1 group was significantly reduced compared with that of the control group (Fig. 2D). These results suggested that uc003pxg.1 promoted cell proliferation and migration.

uc003pxg.1 promotes the expression of cell cycle-associated proteins. uc003pxg.1 expression in HUVECs was knocked down by $\sim 60 \%$ following transfection with si-uc003pxg.1 (Fig. 3A). The RT-qPCR results demonstrated that the expression levels of cyclin D1 and CDK6 were significantly decreased following uc003pxg.1 knockdown compared with those in the control group (Fig. 3B). Western blotting was performed to assess the expression levels of proteins associated with the cell cycle (vimentin, PCNA, CDK4, CDK6, cyclin D1, cyclin B1 and cyclin E1), and the results demonstrated that the levels 


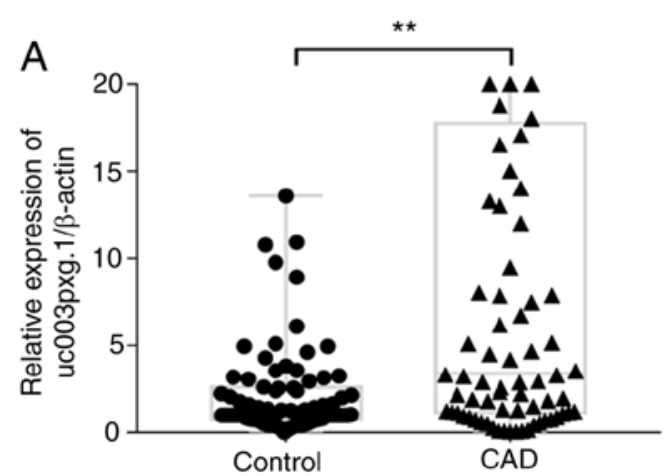

B
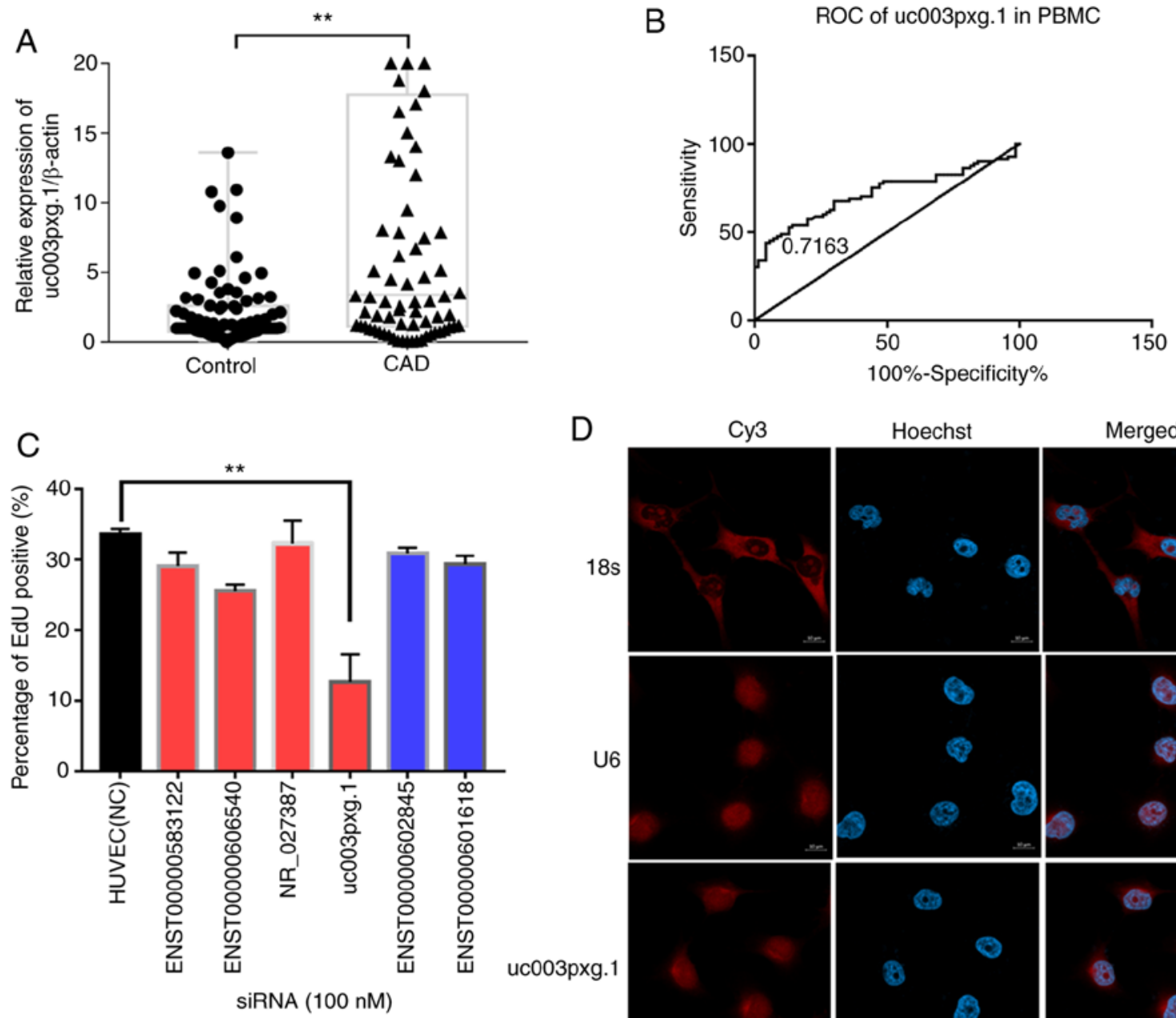

D
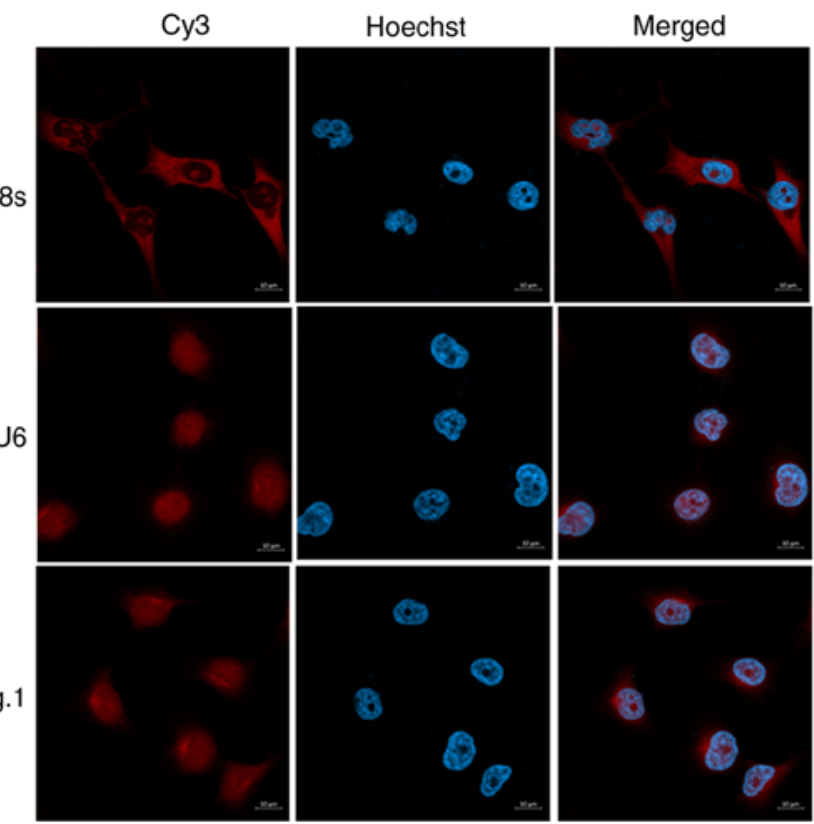

Figure 1. Identification and cell localization of target lncRNAs. (A) The levels of $u c 003 p x g .1$ expression in PBMC samples (n=80). (B) ROC curve of uc003pxg.1 expression in the PBMC samples. (C) Selection of target lncRNAs by high-content screening of uc003pxg.1. Black, NC; red, upregulated lncRNA; blue, downregulated lncRNA. (D) Confocal microscopy images of uc003pxg.1 cellular localization analysis by fluorescence in situ hybridization. ${ }^{* *} \mathrm{P}<0.01$. lncRNA, long noncoding RNA; PBMCs, peripheral blood mononucleated cells; ROC, receiver operating characteristic; CAD, coronary artery disease; NC, negative control; siRNA, small interfering RNA.

of CDK6 and cyclin D1 protein expression were significantly decreased following uc003pxg.1 knockdown compared with those in the control group (Fig. 3C). These results suggested that $u c 003 p x g .1$ promoted the expression of cell cycle-related proteins and that uc003pxg.1 may be associated with the cyclin D1/CDK6 signaling pathway, which is involved in the $\mathrm{G}_{1} / \mathrm{S}$ phase of the cell cycle.

Target miRNAs of uc003pxg.1 identified by high-throughput sequencing. After HUVECs were transfected with siRNA, RNA was extracted for high-throughput sequencing. Differential expression between two sets of samples was calculated, and the most differentially expressed miRNAs (14 upregulated and 5 downregulated) were selected for further analysis (Fig. 4A-C). The results demonstrated that the Ras and MAPK signaling pathways were enriched with the differentially expressed miRNAs (Fig. 4D).

miR-25-5p levels are negatively correlated with those of uc003pxg.1. The expression levels of miRNAs were verified in HUVECs following transfection with si-uc003pxg.1, as well as in PBMCs from clinical samples by RT-qPCR. $m i R-25-5 p$, which is associated with cell proliferation and migration, was selected for further analysis according to previous studies $(29,30)$. The results demonstrated that the expression levels of $m i R-25-5 p$ were significantly upregulated following uc003pxg.1 knockdown compared with those in the control cells (Fig. 5A). The expression levels of $m i R-25-5 p$ were significantly downregulated in samples from 25 patients with CAD compared with those in 25 control samples (Fig. 5B), and $m i R-25-5 p$ expression levels were significantly negatively correlated with those of $u c 003 p x g .1$ in clinical PBMC samples (Fig. 5C). The luciferase activity of the $m i R-25-5 p$ mimic + uc003pxg.1-wt group was significantly decreased compared with that of the NC group, whereas no significant differences in the luciferase activities were observed between the $m i R-25-5 p$ mimic and NC samples in the mut group (Fig. 5D). Taken together, these results suggested that uc003pxg.1 interacted with $m i R-25-5 p$.

miR-25-5p downregulates cyclin D1 and CDK6 expression and inhibits cell proliferation. HUVECs were transfected 
A

EdU

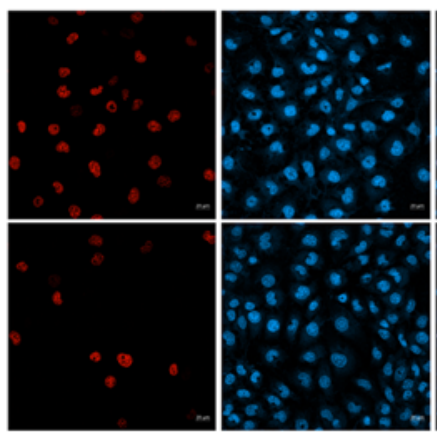

C

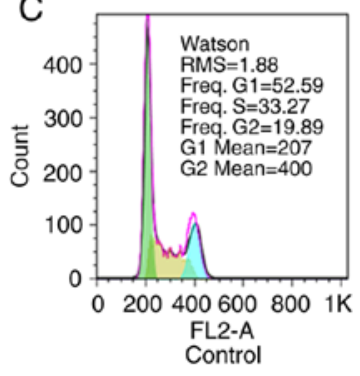

$\mathrm{D}$

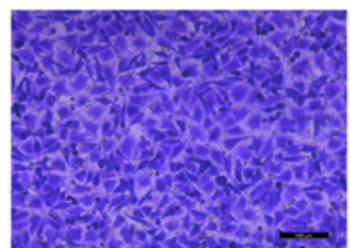

Control
Overlay
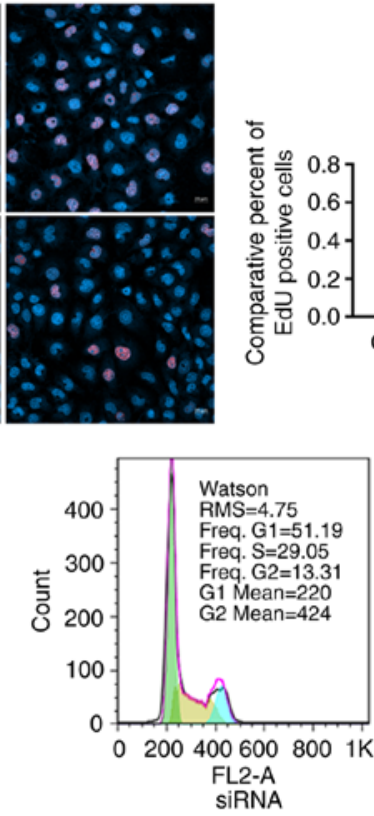

B

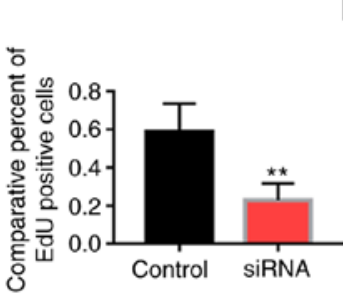

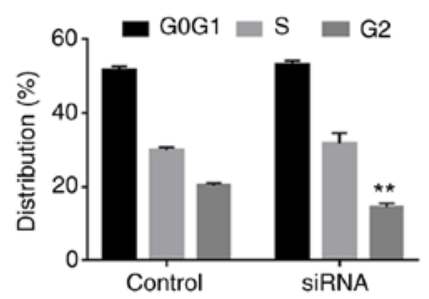

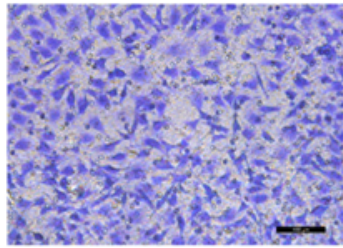

SiRNA

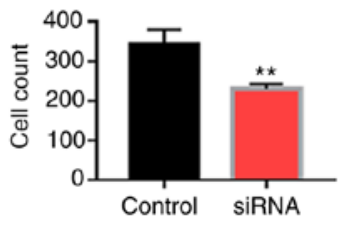

Figure 2. uc003pxg.1 knockdown promotes human umbilical vein endothelial cell proliferation and migration. (A) Confocal microscopy images of EdU staining were used to assess cell proliferation. ${ }^{* *} \mathrm{P}<0.01$. (B) Cell viability was determined by Cell Counting Kit- 8 assay. (C) Flow cytometric analysis of the cell cycle. (D) Migration of the control and siRNA-transfected cells was evaluated by Transwell assay. ${ }^{* *} \mathrm{P}<0.01$ vs. control. EdU, 5-ethynyl-2'-deoxyuridine; control, negative control small interfering RNA; siRNA, small interfering RNA targeting uc003pxg.1.

A

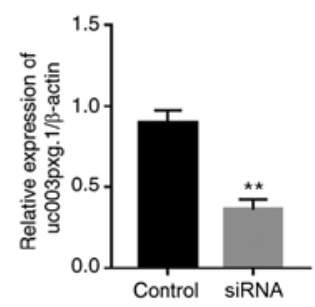

C
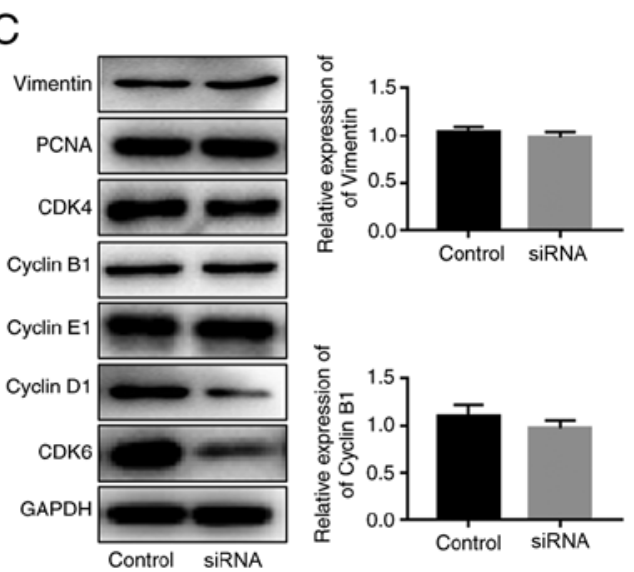
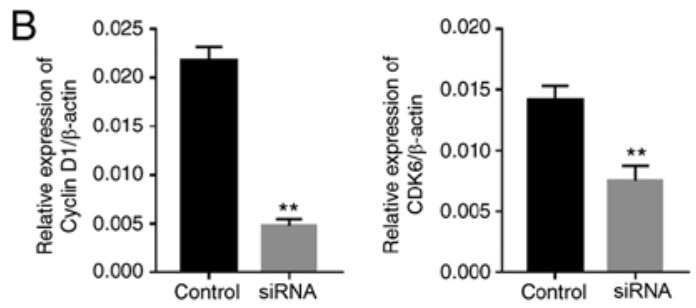
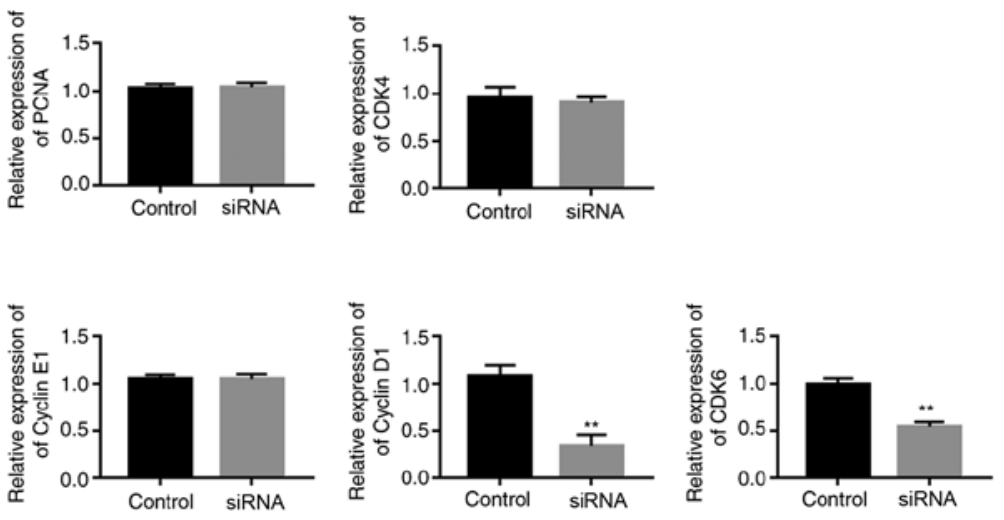

Figure 3. uc003pxg.1 knockdown affects the expression of cell cycle-associated proteins. (A) The knockdown efficiency of si-uc003pxg.1 in HUVECs was detected by RT-qPCR. (B) The mRNA expression levels of cyclin D1 and CDK6 were assessed by RT-qPCR following uc003pxg.1 knockdown in HUVECs (C) The expression levels of cell cycle-associated proteins were detected by western blotting following siRNA transfection. ${ }^{* *} \mathrm{P}<0.01 \mathrm{vs}$. control. HUVECs, human umbilical vein endothelial cells; control, negative control small interfering RNA; siRNA, small interfering RNA targeting $u c 003 p x g .1$; RT-qPCR, reverse transcription-quantitative PCR; PCNA, proliferating cell nuclear antigen. 
A

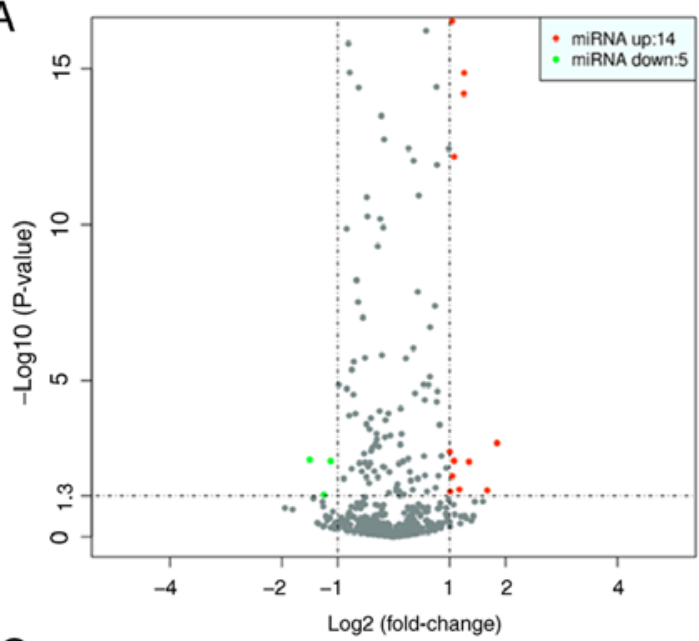

B

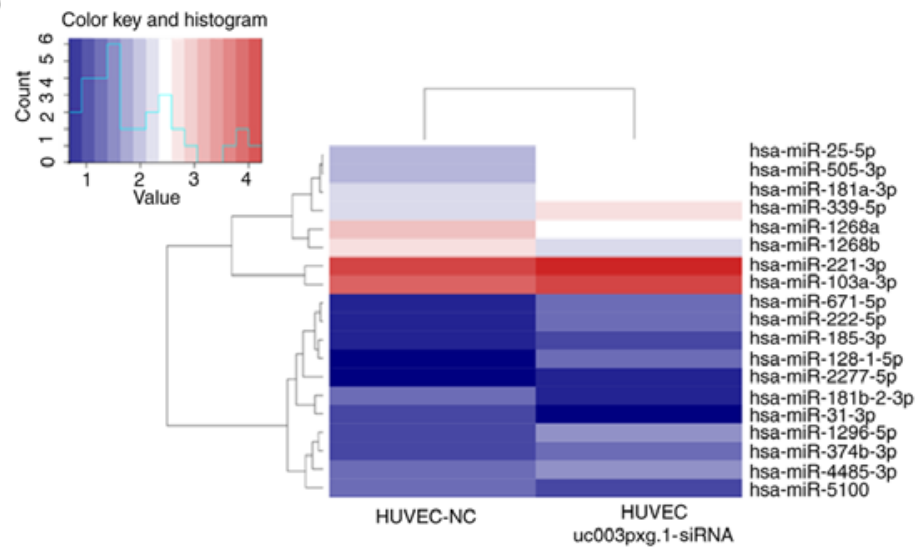

D

C
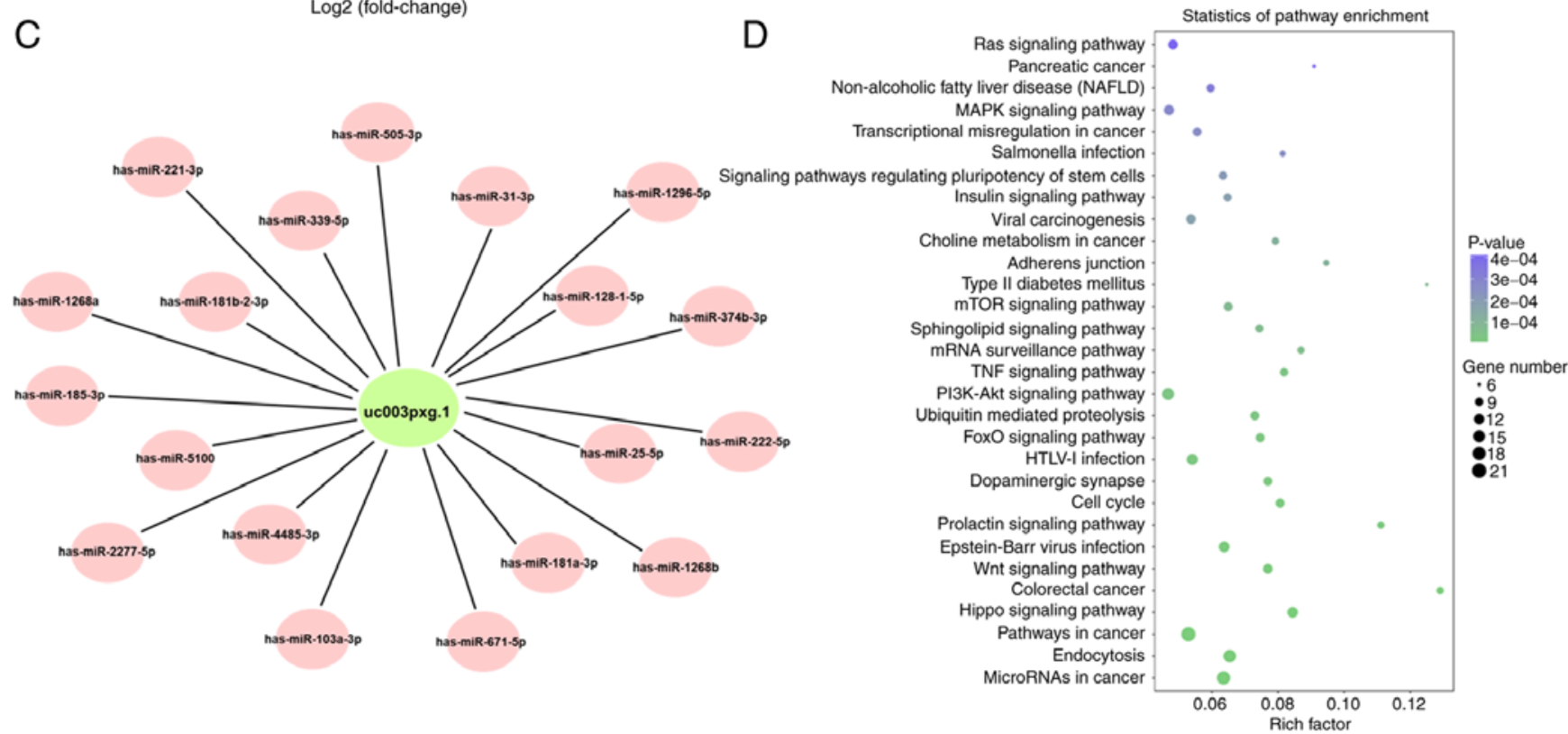

Figure 4. Differentially expressed miRNAs associated with uc003pxg.1 identified by high-throughput sequencing. (A) Differential expression of miRNAs between the control and siRNA-transfected groups was analyzed by a volcano plot. Red, upregulation; green, downregulation. (B) Cluster analysis of the differentially expressed miRNAs between the control and siRNA-transfected groups. (C) Competing endogenous RNA network of $u c 003 p x g .1$ and target miRNAs. (D) Kyoto Encyclopedia of Genes and Genomes pathway analysis of the candidate target genes. miRNA, miR, microRNA; NC, negative control small interfering RNA; siRNA, small interfering RNA targeting uc003pxg.1.

with si-uc003pxg.1, miR-25-5p mimics or $m i R-25-5 p$ inhibitor. The relative expression levels of $m i R-25-5 p$ were significantly increased in HUVECs following transfection with the $m i R-25-5 p$ mimics compared with those in cells transfected with the mimic NC, whereas a miRNA inhibitor that functionally combined the endogenous miRNA competitively and thus regulated the suppression on downstream genes was used, which had no notable effects on the expression levels of $m i R-25-5 p$ (Fig. 6A). Subsequently, cell proliferation was analyzed by the EdU assay. The results demonstrated that the miR-25-5p mimics and si-uc003pxg.1 reduced cell proliferation compared with that in the control group, whereas the $m i R-25-5 p$ inhibitor increased cell proliferation compared with that in the control group, although the difference was not statistically significant (Fig. 6B). In addition, si-uc003pxg.1 and the miR-25-5p mimics inhibited the protein expression levels of cyclin D1 and CDK6 compared with those in the control group (Fig. 6C). These results suggested that $m i R-25-5 p$ exerted the opposite function to uc003pxg.1 in the inhibition of cell proliferation.

miR-25-5p inhibits HUVEC viability, cell cycle and migration. The roles of $m i R-25-5 p$ in cell proliferation and migration were further verified by cell cycle (Fig. 7A), CCK-8 (Fig. 7B) and Transwell migration (Fig. 7C) assays. The CCK-8 and cell cycle assay results demonstrated that cell proliferation was significantly reduced at $48 \mathrm{~h}$ with no difference at $72 \mathrm{~h}$ in the $m i R-25-5 p$ mimic group compared with that in the control group, which was consistent with the results obtained with cells transfected with si-uc003pxg.1. The cell proliferative ability was significantly enhanced in the $m i R-25-5 p$ inhibitor group at $48 \mathrm{~h}$ compared with that in the control group. Furthermore, the Transwell assay results revealed that the $m i R-25-5 p$ mimic and si-uc003pxg.1 significantly reduced cell migration compared 


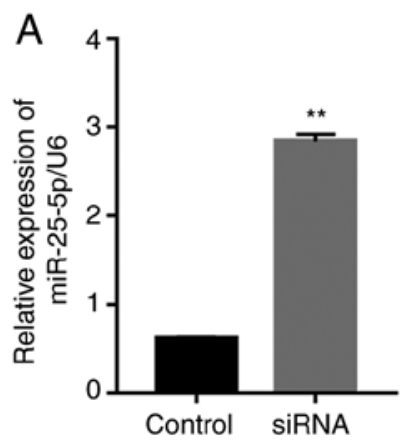

B

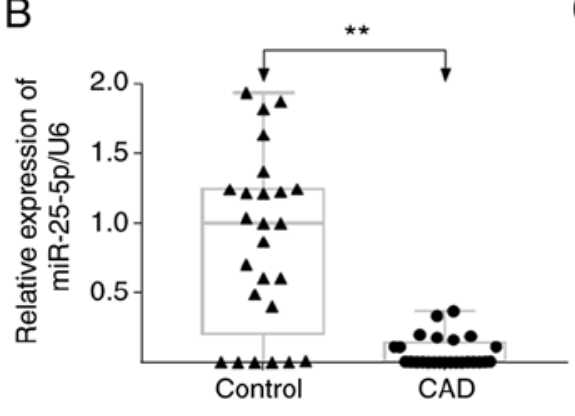

C

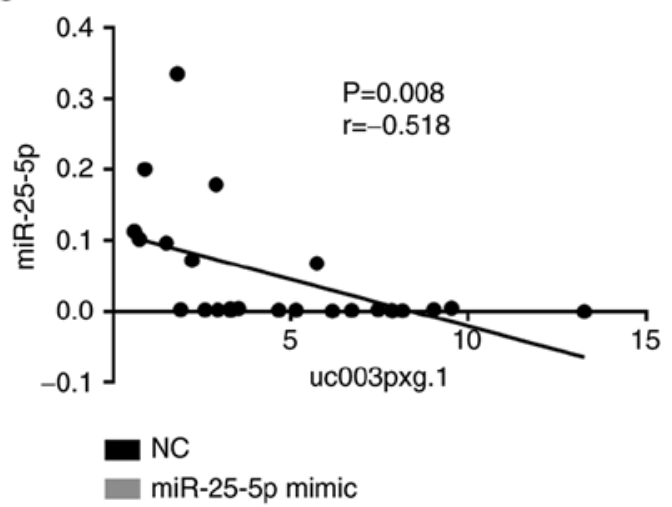

D $\begin{array}{cc}\text { uc003pxg.1 WT } & \text { 5'GCTCACTGCAAG - CTCCGCCt 3' } \\ \text { I I I I I I } \\ \text { hsa-miR-25-5p } & \text { 3'GTTAACGGGTTCAGAGGCGGa 5' } \\ & \\ \text { uc003pxg.1 Mut } & \text { 5'GCTCACTGCAAG - AGAATAAt 3' }\end{array}$

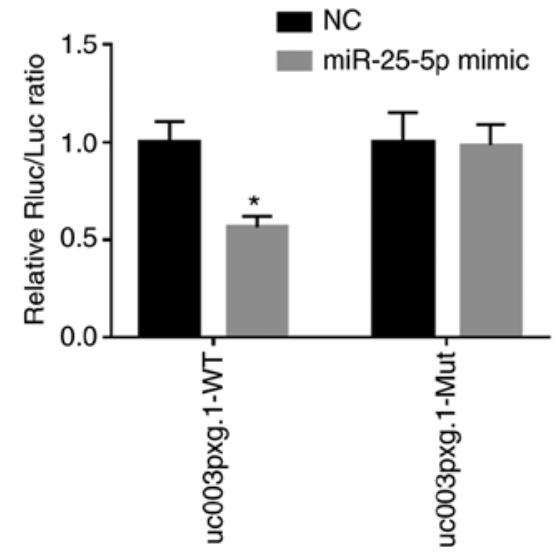

Figure 5. Correlation between $m i R-25-5 p$ and $u c 003 p x g .1$ expression levels. (A) Relative expression levels of $m i R-25-5 p$ in HUVECs in the siRNA and control groups. ${ }^{* *} \mathrm{P}<0.01$ vs. control. (B) Expression levels of $m i R-25-5 p$ in peripheral blood mononucleated cells from patients with $\mathrm{CAD}$ and controls ( $\left.\mathrm{n}=25\right)$. ${ }^{* *} \mathrm{P}<0.01$. (C) $u c 003 p x g .1$ expression levels were negatively correlated with those of $m i R-25-5 p$ in patients with CAD. (D) Luciferase activities were determined in the miR-25-5p mimic + uc003pxg.1-wt and miR-25-5p mimic + uc003pxg.1-mut groups. ${ }^{*}<0.05$ vs. NC. miR, microRNA; HUVECs, human umbilical vein endothelial cells; control, mimic-NC; siRNA, small interfering RNA targeting uc003pxg.1; CAD, coronary artery disease; wt, wild-type; mut, mutant.

with that in the control group, whereas the $m i R-25-5 p$ inhibitor enhanced cell migration compared with that in the control group.

\section{Discussion}

CAD is a chronic inflammatory disease that leads to the formation of medium to large arterial plaques (31). As arterial plaques gradually increase and cause narrowing of the arterial lumen, they can block the blood flow and lead to ischemia of the heart (32). Previous studies have reported that circulating PBMCs, 5-10\% of which are peripheral blood white blood cells, serve important roles in the development of CAD by promoting cell migration to the arterial wall and increasing the extent of atherosclerosis $(33,34)$. Furthermore, lncRNAs have emerged as regulatory factors in the development of atherosclerosis $(12,13)$. Thus, it is necessary to study the differential expression of lncRNAs in patients with CAD to develop noninvasive strategies for the early diagnosis of CAD.

In our previous study, a number of lncRNAs were identified as differentially expressed in patients with CAD compared with healthy controls through high-throughput sequencing (22). Among these lncRNAs, the functional lncRNA uc003pxg.l was demonstrated to function in cell proliferation and migration through high-content analysis in the present study. Clinical samples from 80 patients with CAD and 80 control subjects were evaluated, and the results revealed that the expression levels of uc003pxg.l were significantly upregulated in patients with CAD compared with those in the control subjects, with the area under the ROC curve of 0.7163 , suggesting that uc003pxg.1 may be a potential biomarker for clinical CAD diagnosis. In addition, the results of the EdU, CCK-8 and cell cycle assays in HUVECs demonstrated that cell proliferation was significantly reduced following uc003pxg.1 knockdown. Transwell experiments revealed a reduction in the cell migratory ability after HUVECs were transfected with si-uc003pxg.1. Taken together, these results suggested that uc003pxg.1 may promote cell proliferation and migration. Additionally, analysis of the expression of cell cycle-related genes and proteins demonstrated that cyclin D1 and CDK6 levels were significantly downregulated after uc003pxg.1 knockdown, further suggesting that uc003pxg.l may be associated with the cell cycle and promote cell proliferation.

The present study also identified differentially expressed miRNAs in si-uc003pxg.1-transfected and control HUVECs via sequencing. The results demonstrated that 14 miRNAs were upregulated and 5 were downregulated following transfection. Among them, the levels of $m i R-25-5 p$ were significantly upregulated in HUVECs following uc003Pxg.l knockdown, but significantly downregulated in clinical CAD samples compared with those in the control samples, demonstrating a negative correlation between miR-25-5p and uc003pxg.1. $\mathrm{miR}-25-5 \mathrm{p}$ has been reported to be involved in cell proliferation and migration in various diseases. For example, miR-25 inhibits the proliferation and cell cycle progression of non-small cell lung cancer cells by targeting cell division 
A
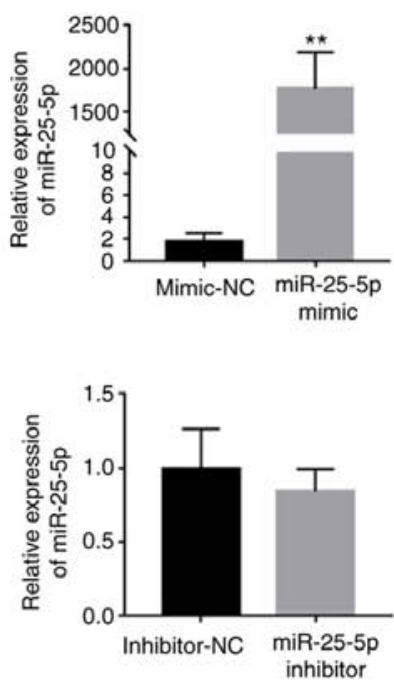

B

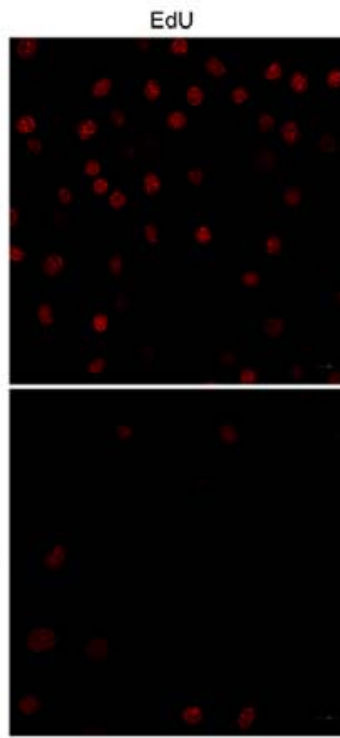

Hoechst

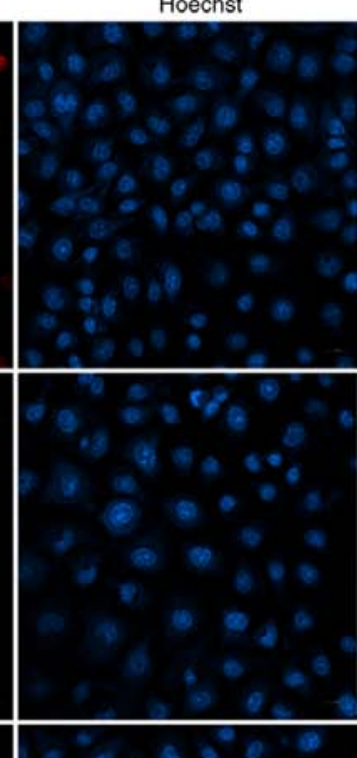

Overlay

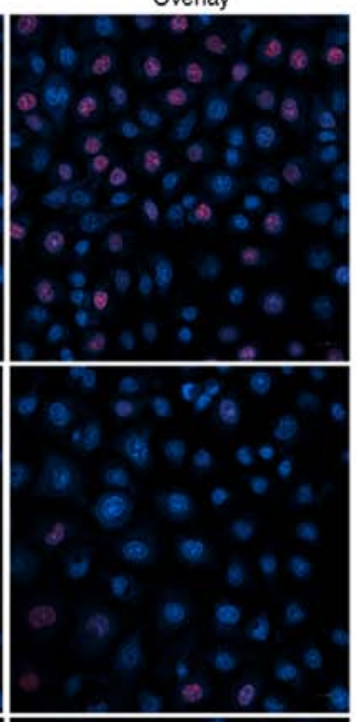

miR-25-5p mimic
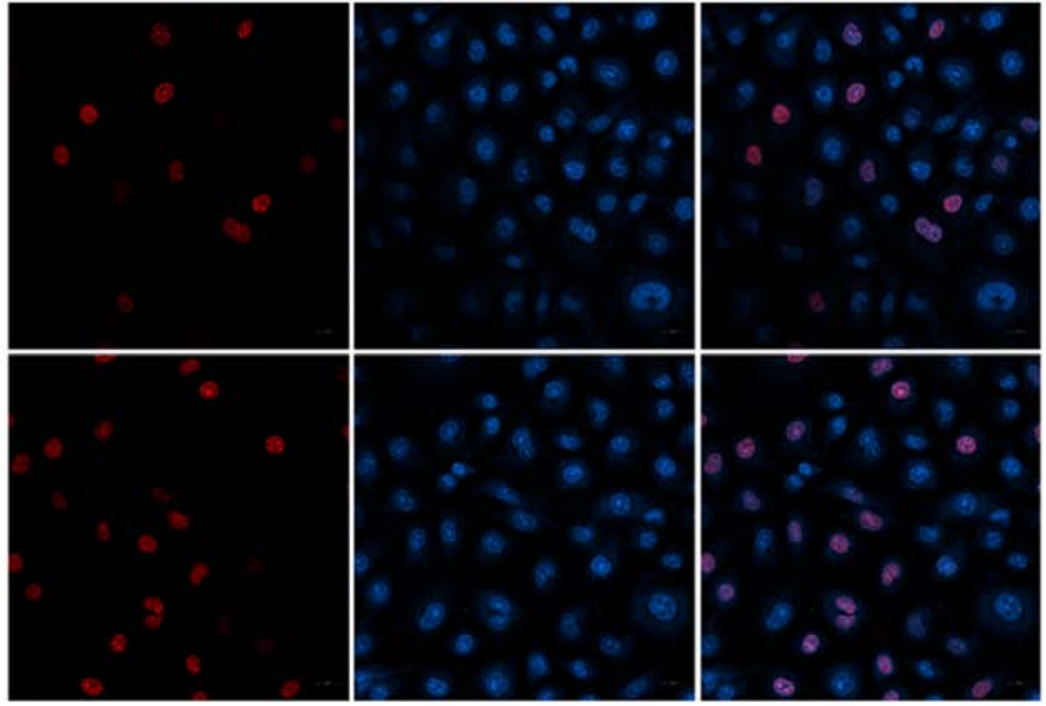

C

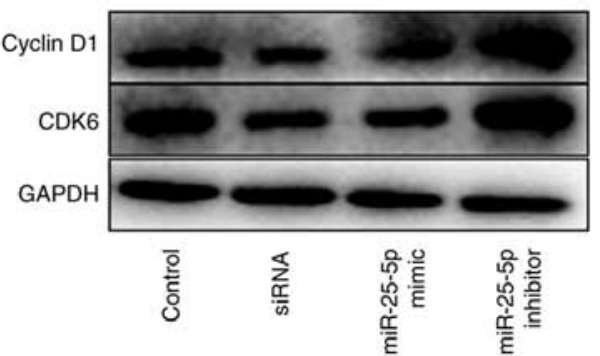

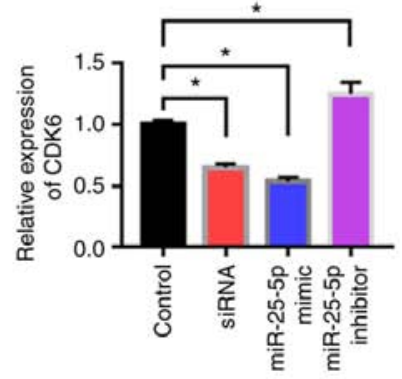

Figure 6. Effects of $u c 003 p x g .1$ and $m i R-25-5 p$ on cell proliferation and related protein expression. (A) The relative expression of $m i R-25-5 p$ in HUVECs after transfection with the miR-25-5p mimics, mimic-NC, miR-25-5p inhibitor and inhibitor-NC. ${ }^{* *} \mathrm{P}<0.01 \mathrm{vs}$. mimics-NC. (B) The association between $m i R-25-5 p$ levels and cell proliferation was analyzed by the EdU assay. Red, EdU; blue, nuclear staining. ${ }^{* *} \mathrm{P}<0.01$. (C) Cyclin D1 and CDK6 expression was determined in HUVECs after transfection with mimic-NC, siRNA, $m i R-25-5 p$ mimic and $m i R-25-5 p$ inhibitor. ${ }^{*} \mathrm{P}<0.05$ and ${ }^{* *} \mathrm{P}<0.01$. miR, microRNA; HUVECs, human umbilical vein endothelial cells; NC, negative control; EdU, 5-ethynyl-2'-deoxyuridine; control, mimic-NC; siRNA, small interfering RNA targeting uc003pxg.1.

cycle 42 expression (35) and also enhances the proliferation, invasion and migration of liver cancer cells by inhibiting Rho GDP dissociation inhibitor $\alpha$ expression (36). Furthermore, mir-25-5p regulates the proliferation, migration and apoptosis of oxidized low-density lipoprotein-treated human brain microvessel endothelial cells by targeting neuronal growth regulator 1 (30), whereas treatment with melatonin and pterostilbene has been demonstrated to promote the apoptosis of colon cancer cells through $m i R-25-5 p$ (37). The combination of PBMC miR-19b-5p, miR-221, miR-25-5p and hypertension is associated with an increased heart failure risk in patients with coronary heart disease (38). In another study, the expression levels of $m i R-25-3 p$ and $m i R-25-5 p$ in the myocardium of rats with heart failure have been reported to be upregulated (39). 

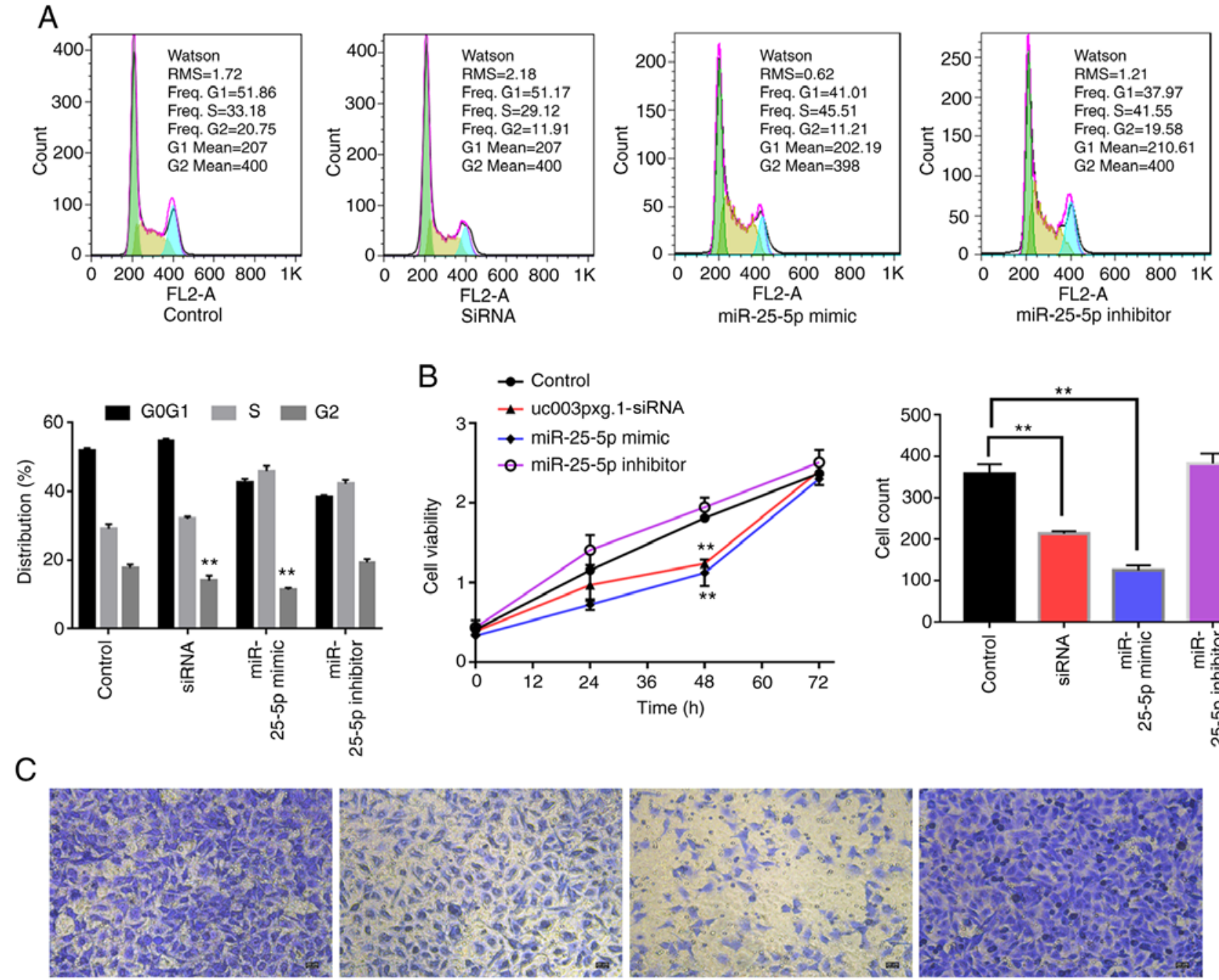

Control

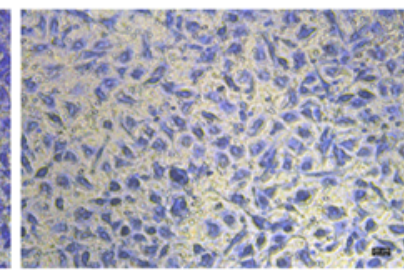

siRNA

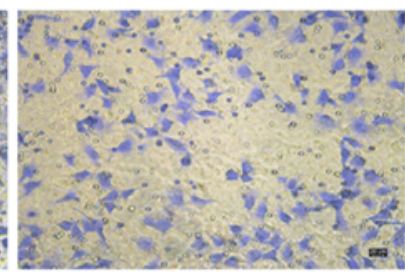

miR-25-5p mimic
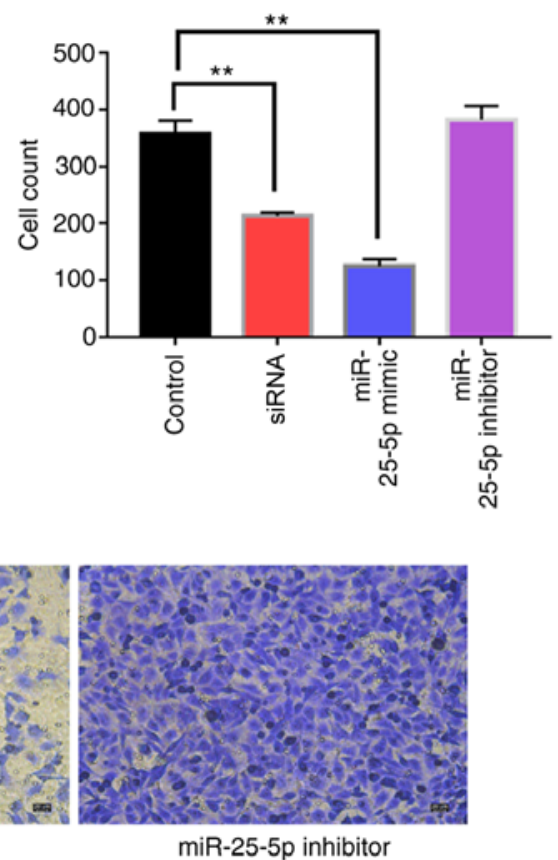

Figure 7. Association between uc003pxg.1 and miR-25-5p during cell proliferation and migration. (A) The cell cycle progression of HUVECs transfected with mimic-NC, siRNA, miR-25-5p mimic and $m i R-25-5 p$ inhibitor was analyzed by flow cytometry. (B) The effects of HUVEC transfection with siRNA, $m i R-25-5 p$ mimic and $m i R-25-5 p$ inhibitor on cell proliferation were analyzed by Cell Counting Kit- 8 assay. (C) The migration of HUVECs transfected with mimic-NC, siRNA, miR-25-5p mimic and $m i R-25-5 p$ was analyzed by Transwell assay. ${ }^{* *} \mathrm{P}<0.01$. miR, microRNA; HUVECs, human umbilical vein endothelial cells; NC, negative control; control, mimic-NC; siRNA, small interfering RNA targeting uc003pxg.1.

In the present study, the effects of $m i R-25-5 p$ on cell proliferation and migration were also evaluated. The EdU, cell cycle and CCK-8 assay results demonstrated that the $m i R-25-5 p$ mimics exerted similar effects to those of si-uc003pxg.1 and significantly reduced the proliferation of HUVECs, whereas the miR-25-5p inhibitor promoted cell proliferation. Transwell assay results also revealed that the $m i R-25-5 p$ mimic, similar to si-uc003pxg.l, reduced the migration of HUVECs, whereas the $m i R-25-5 p$ inhibitor promoted the migratory ability of HUVECs.

Due to their cell and tissue specificity, IncRNAs can be used as cancer biomarkers and therapeutic targets (10). lncRNAs are secreted or released from necrotic and apoptotic cells in the blood and urine (40), and have been demonstrated to serve important roles in inflammation, vascular smooth muscle cell proliferation, apoptosis and fat metabolism $(13,41)$. IncRNAs are also associated with the occurrence and development of CAD (42). The results of the present study revealed that the levels of IncRNA uc003pxg.l were significantly upregulated in patients with CAD compared with those in the control subjects, and that uc003pxg.1 promoted cell proliferation, migration and cell cycle-related gene and protein (cyclin D1 and CDK6) expression. In addition, an interaction between $m i R-25-5 p$ and $u c 003 p x g .1$ was identified through high-throughput sequencing and dual-luciferase reporter gene assays. For the first time, the present study demonstrated that uc003pxg.1-targeted $m i R-25-5 p$ regulates cell proliferation and migration. The high expression levels of uc003pxg.1 in circulating peripheral blood cells of patients with CAD may increase the proliferation and migration of vascular endothelial cells and promote the development of CAD by sponging $m i R-25-5 \mathrm{p}$. These results may provide a new lncRNA target in the study of CAD and a potential biomarker for the early diagnosis of CAD. However, to confirm these findings, a larger number of clinical samples, additional in vivo experiments, including those to assess the role of uc003pxg.l in inflammation and other pathways, need to be performed, which will be carried out in our future study. 


\section{Acknowledgements}

Not applicable.

\section{Funding}

This work was supported by the Special Diagnosis Techniques for Clinical Key Diseases of Suzhou Municipal Health and Family Planning Commission (grant no. LCZX201910), the Science and Technology Development Fund of Nanjing Medical University (grant no. NMUB2018219), The Natural Science Foundation of Jiangsu Province for Youth (grant no. BK20190189) and the Youth Program of National Natural Science Foundation of China (grant no. 82000379).

\section{Availability of data and materials}

The datasets used and/or analyzed during the current study are available from the corresponding author on reasonable request.

\section{Authors' contributions}

KS and GX designed the study and obtained funding. PL performed the experiments and drafted the manuscript. YL, LC and XM collected and interpretated the data. XY, MY, BQ, FW, JX and JY conducted literature search and data interpretation. All authors reviewed the results. All authors read and approved the final manuscript. XY and JY confirm the authenticity of all the raw data.

\section{Ethics approval and consent to participate}

The study was approved by the Ethics Committee of Nanjing Medical University (KL901117). Informed consent was obtained from the patients and their families.

\section{Patient consent for publication}

Not applicable.

\section{Competing interests}

The authors declare that they have no competing interests.

\section{References}

1. Huang L, Zheng Y, Yuan X, Ma Y, Xie G, Wang W, Chen H and Shen L: Decreased frequencies and impaired functions of the $\mathrm{CD} 31^{+}$subpopulation in $\mathrm{T}_{\text {reg }}$ cells associated with decreased FoxP3 expression and enhanced $\mathrm{T}_{\mathrm{reg}}$ cell defects in patients with coronary heart disease. Clin Exp Immunol 187: 441-454, 2017.

2. Jokinen E: Coronary artery disease in patients with congenital heart defects. J Intern Med 288: 383-389, 2020.

3. Malakar AK, Choudhury D, Halder B, Paul P, Uddin A and Chakraborty $\mathrm{S}$ : A review on coronary artery disease, its risk factors, and therapeutics. J Cell Physiol 234: 16812-16823, 2019.

4. Valgimigli M, Bueno $\mathrm{H}$, Byrne RA, Collet JP, Costa F, Jeppsson A, Jüni P, Kastrati A, Kolh P, Mauri L, et al: 2017 ESC focused update on dual antiplatelet therapy in coronary artery disease developed in collaboration with EACTS: The Task Force for dual antiplatelet therapy in coronary artery disease of the European Society of Cardiology (ESC) and of the European association for cardio-thoracic surgery (EACTS). Eur Heart J 39: 213-260, 2018
5. Cai Y, Yang Y, Chen X, Wu G, Zhang X, Liu Y, Yu J, Wang X, Fu J, Li C, et al: Circulating 'IncRNA OTTHUMT00000387022' from monocytes as a novel biomarker for coronary artery disease. Cardiovasc Res 112: 714-724, 2016.

6. Huang Y: The novel regulatory role of lncRNA-miRNA-mRNA axis in cardiovascular diseases. J Cell Mol Med 22: 5768-5775, 2018.

7. Bhan A and Mandal SS: LncRNA HOTAIR: A master regulator of chromatin dynamics and cancer. Biochim Biophys Acta 1856: $151-164,2015$.

8. Derrien T, Johnson R, Bussotti G, Tanzer A, Djebali S, Tilgner H, Guernec G, Martin D, Merkel A, Knowles DG, et al: The GENCODE v7 catalog of human long noncoding RNAs: Analysis of their gene structure, evolution, and expression. Genome Res 22: 1775-1789, 2012.

9. Sanfilippo PG and Hewitt AW: Translating the ENCyclopedia Of DNA elements project findings to the clinic: ENCODE's implications for eye disease. Clin Exp Ophthalmol 42: 78-83, 2014.

10. Bhan A, Soleimani M and Mandal SS: Long noncoding RNA and cancer: A new paradigm. Cancer Res 77: 3965-3981, 2017.

11. Kaikkonen MU, Lam MT and Glass CK: Non-coding RNAs as regulators of gene expression and epigenetics. Cardiovasc Res 90: 430-440, 2011.

12. Greco S, Gorospe M and Martelli F: Noncoding RNA in age-related cardiovascular diseases. J Mol Cell Cardiol 83: $142-155,2015$.

13. Li L, Wang L, Li H, Han X, Chen S, Yang B, Hu Z, Zhu H, Cai C, Chen J, et al: Characterization of LncRNA expression profile and identification of novel LncRNA biomarkers to diagnose coronary artery disease. Atherosclerosis 275: 359-367, 2018.

14. Zhang H, Ji N, Gong X, Ni S and Wang Y: NEAT1/miR140-3p/MAPK1 mediates the viability and survival of coronary endothelial cells and affects coronary atherosclerotic heart disease. Acta Biochim Biophys Sin (Shanghai) 52: 967-974, 2020.

15. Zhang Z, Gao W, Long QQ, Zhang J, Li YF, Liu DC, Yan JJ, Yang ZJ and Wang LS: Increased plasma levels of lncRNA H19 and LIPCAR are associated with increased risk of coronary artery disease in a Chinese population. Sci Rep 7: 7491, 2017.

16. Tang Y, Jin X, Xiang Y, Chen Y, Shen CX, Zhang YC and Li YG: The lncRNA MALAT1 protects the endothelium against ox-LDL-induced dysfunction via upregulating the expression of the miR-22-3p target genes CXCR2 and AKT. FEBS Lett 589: 3189-3196, 2015.

17. Michalik KM, You X, Manavski Y, Doddaballapur A, Zörnig M, Braun T, John D, Ponomareva Y, Chen W, Uchida S, et al: Long noncoding RNA MALAT1 regulates endothelial cell function and vessel growth. Circ Res 114: 1389-1397, 2014.

18. Motterle A, Pu X, Wood H, Xiao Q, Gor S, Ng FL, Chan K, Cross F, Shohreh B, Poston RN, et al: Functional analyses of coronary artery disease associated variation on chromosome 9 p21 in vascular smooth muscle cells. Hum Mol Genet 21: 4021-4029, 2012.

19. Ivanov D, Philippova M, Allenspach R, Erne P and Resink T: T-cadherin upregulation correlates with cell-cycle progression and promotes proliferation of vascular cells. Cardiovasc Res 64: 132-143, 2004.

20. Wang G, Li Y, Peng Y, Tang J and Li H: Association of polymorphisms in MALAT1 with risk of coronary atherosclerotic heart disease in a Chinese population. Lipids Health Dis 17: 75, 2018.

21. Wu Z, He Y, Li D, Fang X, Shang T, Zhang H and Zheng X: Long noncoding RNA MEG3 suppressed endothelial cell proliferation and migration through regulating miR-21. Am J Transl Res 9: 3326-3335, 2017.

22. Li P, Yan X, Xu G, Pang Z, Weng J, Yin J, Li M, Yu L, Chen Q and Sun K: A novel plasma lncRNA ENST00000416361 is upregulated in coronary artery disease and is related to inflammation and lipid metabolism. Mol Med Rep 21: 2375-2384, 2020.

23. Wenger NK: 2011 ACCF/AHA focused update of the guidelines for the management of patients with unstable angina/Non-ST-elevation myocardial infarction (updating the 2007 guideline): Highlights for the clinician. Clin Cardiol 35: $3-8,2012$.

24. Jia Y, Xu H, Li Y, Wei C, Guo R, Wang F, Wu Y, Liu J, Jia J, Yan J, et al: A modified ficoll-paque gradient method for isolating mononuclear cells from the peripheral and umbilical cord blood of humans for biobanks and clinical laboratories. Biopreserv Biobank 16: 82-91, 2018.

25. Trapnell C, Hendrickson DG, Sauvageau M, Goff L, Rinn JL and Pachter L: Differential analysis of gene regulation at transcript resolution with RNA-seq. Nat Biotechnol 31: 46-53, 2013. 
26. Livak KJ and Schmittgen TD: Analysis of relative gene expression data using real-time quantitative PCR and the 2(-Delta Delta C(T)) method. Methods 25: 402-408, 2001

27. Wang L, Feng Z, Wang X, Wang X and Zhang X: DEGseq: An $\mathrm{R}$ package for identifying differentially expressed genes from RNA-seq data. Bioinformatics 26: 136-138, 2010.

28. Liu H, Lei C, He Q, Pan Z, Xiao D and Tao Y: Nuclear functions of mammalian MicroRNAs in gene regulation, immunity and cancer. Mol Cancer 17: 64, 2018

29. Wang Y, Tao B, Li J, Mao X, He W and Chen Q: Melatonin inhibits the progression of oral squamous cell carcinoma via inducing miR-25-5p expression by directly targeting NEDD9. Front Oncol 10: 543591,2020.

30. Zhang Q, Liu C, Li Q, Li J, Wu Y and Liu J: MicroRNA-25-5p counteracts oxidized LDL-induced pathological changes by targeting neuronal growth regulator 1 (NEGR1) in human brain micro-vessel endothelial cells. Biochimie 165: 141-149, 2019.

31. Niccoli G, Montone RA, Sabato V and Crea F: Role of allergic inflammatory cells in coronary artery disease. Circulation 138 $1736-1748,2018$

32. Weber $\mathrm{C}$ and Noels $\mathrm{H}$ : Atherosclerosis: Current pathogenesis and therapeutic options. Nat Med 17: 1410-1422, 2011.

33. Knorr M, Münzel T and Wenzel P: Interplay of NK cells and monocytes in vascular inflammation and myocardial infarction. Front Physiol 5: 295, 2014.

34. Jaipersad AS, Shantsila A, Lip GY and Shantsila E: Expression of monocyte subsets and angiogenic markers in relation to carotid plaque neovascularization in patients with pre-existing coronary artery disease and carotid stenosis. Ann Med 46: 530-538, 2014.

35. Yang T, Chen T, Li Y, Gao L, Zhang S, Wang T and Chen M: Downregulation of miR-25 modulates non-small cell lung cancer cells by targeting CDC42. Tumour Biol 36: 1903-1911, 2015.
36. Wang C, Wang X, Su Z, Fei H, Liu X and Pan Q: MiR-25 promotes hepatocellular carcinoma cell growth, migration and invasion by inhibiting RhoGDI1. Oncotarget 6: 36231-36244, 2015.

37. Jung JH, Shin EA, Kim JH, Sim DY, Lee H, Park JE, Lee HJ and Kim SH: NEDD9 inhibition by miR-25-5p activation is critically involved in co-treatment of melatonin- and pterostilbene-induced apoptosis in colorectal cancer cells. Cancers (Basel) 11: 1684, 2019.

38. Yao Y, Song T, Xiong G, Wu Z, Li Q, Xia H and Jiang X: Combination of peripheral blood mononuclear cell miR-19b-5p, miR-221, miR-25-5p, and hypertension correlates with an increased heart failure risk in coronary heart disease patients. Anatol J Cardiol 20: 100-109, 2018.

39. Wu JB, Ye XH, Xian SX and Dong MG: Expressions of SERCA2a and $\mathrm{miR}-25-3 \mathrm{p} / 5 \mathrm{p}$ in myocardium of rats with heart failure and therapeutic effects of Xiefei Lishui recipe. Zhongguo Ying Yong Sheng Li Xue Za Zhi 33: 146-150, 2017 (In Chinese).

40. Meseure D, Drak Alsibai K, Nicolas A, Bieche I and Morillon A: Long noncoding RNAs as new architects in cancer epigenetics, prognostic biomarkers, and potential therapeutic targets. Biomed Res Int 2015: 320214, 2015.

41. Rizzacasa B, Amati F, Romeo F, Novelli G and Mehta JL: Epigenetic modification in coronary atherosclerosis: JACC review topic of the week. J Am Coll Cardiol 74: 1352-1365, 2019.

42. Zhang YH, Pan X, Zeng T, Chen L, Huang T and Cai YD: Identifying the RNA signatures of coronary artery disease from combined IncRNA and mRNA expression profiles. Genomics 112: 4945-4958, 2020.

(i) $\odot$ This work is licensed under a Creative Commons Attribution-NonCommercial-NoDerivatives 4.0 International (CC BY-NC-ND 4.0) License. 